\title{
Five-year follow-up study of stage I-IV rectal cancer including EGFR immunoexpression and p21 immunoactivity
}

\author{
Monika Kozłowska-Geller ${ }^{1}$, Stanisław Głuszek ${ }^{2}$, Piotr Lewitowicz ${ }^{3}$ \\ ${ }^{1}$ Department of Physiology, Institute of Medical Science, Collegium Medicum, Jan Kochanowski University, Kielce, Poland \\ 2Department of Surgery and Surgical Nursing, Collegium Medicum, Jan Kochanowski University, Kielce, Poland \\ ${ }^{3}$ Department of Pathology, Collegium Medicum, Jan Kochanowski University, Kielce, Poland
}

Gastroenterology Rev 2021; 16 (4): 330-338 DOI: https://doi.org/10.5114/pg.2021.104980

Key words: rectal cancer, follow-up, EGFR, P21.

Address for correspondence: Monika Kozłowska-Geller MD, PhD, Collegium Medicum, Jan Kochanowski University, Al. IX Wieków Kielc 19a, 25-317 Kielce, Poland, phone: +48 4134969 01/09, fax: +48 4134969 16, e-mail: monika.kozlowska.chir@onet.pl

\begin{abstract}
Introduction: Both environmental and genetic factors increase the likelihood of developing rectal cancer.

Aim: To assess the EGFR and p21 immunoreactivity in rectal cancer and to assess its relationship with the clinical outcome.

Material and methods: Applying exclusion criteria, 102 patients with stage I-IV rectal cancer, who had undergone scheduled surgery during the period 2005-2011, were included in the study. There was a follow-up study with a span of 5 years from the date of the surgery. Immunohistochemistry using epidermal growth factor receptor (EGFR Ab10, Clone111.6) and antibodies against p21 (p2 $1^{\text {WAF1 }}$ (Clone H252)) was performed to detect overexpression of the targeted receptor. Digital analysis of positive reactions of membranes and nuclei was performed utilizing Visiopharm.

Results: The degree of EGFR intensity ( $\log \mathrm{OR}=0.854, \mathrm{OR}=2.35,95 \% \mathrm{Cl}: 1.14-4.85, p=0.021)$ is a significant factor in the prognosis of death within 2 years after surgery. The OS curve showed a significant decrease after 40 months from the date of surgery in the cases where EGFR had high expression. The ROC curve for cancer stage, according to the UICC classification and EGFR expression, in order to predict 2-year RFS, reached a high specificity value ( $R O C=0.81, p=0.0408$ ). The analysis showed no statistically significant differences in the survival curves of patients in groups with immunoreactivity of p21 protein at 0 , $1,2,3(p=0.6453$ in the log-rank test). Also, it is not a significant risk factor for death ( $H R=0.915, p=0.7842)$ or for tumor dissemination ( $\mathrm{HR}=0.94, p=0.9426$ ).

Conclusions: The determination of EGFR immunoreactivity is important in the monitoring and treatment of patients with rectal cancer, as opposed to p21.
\end{abstract}

\section{Introduction}

Rectal cancer is the third leading cancer worldwide and the fourth cause of death. It is known that both environmental and genetic factors increase the likelihood of developing it. Most rectal cancers are sporadic. Adenocarcinomas account for $95 \%$ of all rectum cancer cases. Treatment of rectal cancer usually involves surgical resection following radiation therapy [1-3]. The survival of rectal cancer patients strongly depends on the clinical stage [4].

The EGFR receptor specific for epidermal growth factor cells (c-ErbB-1/HER), is a member of the transmembrane class 1 receptors containing two cyste- ine-rich domains in the extracellular part, and in the intra-plasmatic part a domain showing activity of tyrosine kinase activity $[4,5]$. The binding of EGF or another ligand to EGFR causes phosphorylation and conformational change with tyrosine kinase activation in the endoplasmic domain. Under normal circumstances, EGF stimulates the proliferation of both mature epithelial cells and stem cells to renew the damaged epithelium. Nevertheless, if uncontrolled, it could lead to cancer [1-3]. It is believed that the pathway, via the RAS protein family, is the key factor in initiating the process of cell proliferation. The normal bowel mucosa does not express EGFR at a detectable level for immunohistochemistry (IHC). Any type of IHC expression proves 
the mutation and amplification of the EGFR gene and over-expression of that signaling pathway [6-8]. In the case of rectal cancer, EGFR over-expression is found in approximately $25-82 \%$ of cases. The intensity of EGFR expression correlates with IHC intensity, the severity of illness and the risk of distant metastases [9]. EGFR triggers secondary transmitters, including KRAS, BRAF and MYC, whilst simultaneously activating the PI3K pro-metabolic pathway [10]. Moreover, a parallel target is the MAPK pathway influencing cell survival. The occurrence of a mutation in the KRAS or BRAF gene means that these proteins are constantly active regardless of the activation or non-activation of EGFR.

The P21 protein is encoded by the WAF1/CIP1 suppressor gene, induced by oncoprotein $\mathrm{p} 53$, a wild-type TP53 gene product, and is involved in inhibiting the cell cycle between $\mathrm{G} 1 / \mathrm{S}$ phases. Its contribution to the regulation of DNA repair and replication, as well as apoptosis modulation, has been proven [1-3]. After DNA damage, p21 induced by p53 either leads to cell cycle arrest or it can evoke cell apoptosis [11]. The involvement of p21 in intracellular transcription of signals from the growth factor receptors to the cell nucleus by activation of the kinase cascade and Raf proto-oncogene has also been described [6-8]. Another p21 function is to induce cell growth arrest by blocking the nuclear antigen of proliferating cell (PCNA) activity in DNA replication and 'mismatch' in DNA repair [12-14]. Therefore, we decided to test the usefulness of p21 as a cancer biomarker.

\section{Aim}

The aim of the study was to assess the survival rate of patients after stage I-IV rectal cancer surgery during the 5-year period after the surgery, including the clinical features, EGFR immunoreactivity, the p21 immunoactivity and their impact on survival without local recurrence, survival with local recurrence and dissemination of cancer.

\section{Material and methods}

Using the appropriate inclusion and exclusion criteria, 102 patients with stage I-IV rectal cancer, who had been operated on between 2005 and 2011, qualified for the study. Inclusion criteria included male and female patients who had undergone scheduled surgery due to cancer of the rectum, in whom metastatic cancer was excluded and who did not have other gastrointestinal tract tumors. These patients qualified for surgery with the intention of being treated, and otherwise suffered from no medical conditions (internal, cardiological, pulmonary). The clinical outcomes were overall survival (OS) and relapse-free survival (RFS), which was understood as local recurrence-free survival and dissemination-free survival.
The clinical features of the patients participating in the study were analyzed, taking into account age, sex, symptoms of the disease, body mass index (BMI), review of preoperative diagnostics, analysis of surgery, postoperative course, especially the occurrence of general and local complications, analysis of histopathological examination of postoperative samples and immunohistochemical tests determining EGFR overexpression and immunoreactivity of p21protein. The second stage of the research was a follow-up that allowed assessment of the patient's survival without local recurrence, with local recurrence and neoplastic spread within 5 years after surgery.

The research methodology was divided into two stages. The first stage included a retrospective analysis of the medical history of patients who qualified for the study. The second stage included immunohistochemical studies assessing EGFR immunoreactivity in postoperative specimens. The study included patients without pre-operative radiotherapy. Finally, 102 patients, aged 41-87, were enrolled in the study. These included 41 women and 61 men with rectal cancer confirmed by a histopathological examination. There was a follow-up study with a span of 5 years from the date of the surgery. All the patients with adjuvant pre-operative radiotherapy were excluded, and all the participants with stage III-IV rectal cancer were subjected to FOLFOX-4 based chemotherapy (oxaliplatin $85 \mathrm{mg} / \mathrm{m}^{2}$, calcium folinate $200 \mathrm{mg} / \mathrm{m}^{2}$, fluorouracil $400 \mathrm{mg} / \mathrm{m}^{2}$, fluorouracil $600 \mathrm{mg} / \mathrm{m}^{2}$ ). The patients' written consent was obtained.

Classic immunohistochemical tests, using an antiEGFR antibody (EGFR 96 Ab10, clone 111.6), were performed. All the tests performed had been fully validated with the intention of in vitro use. All the reactions were carried out using BenchMark XT (Ventana Medical

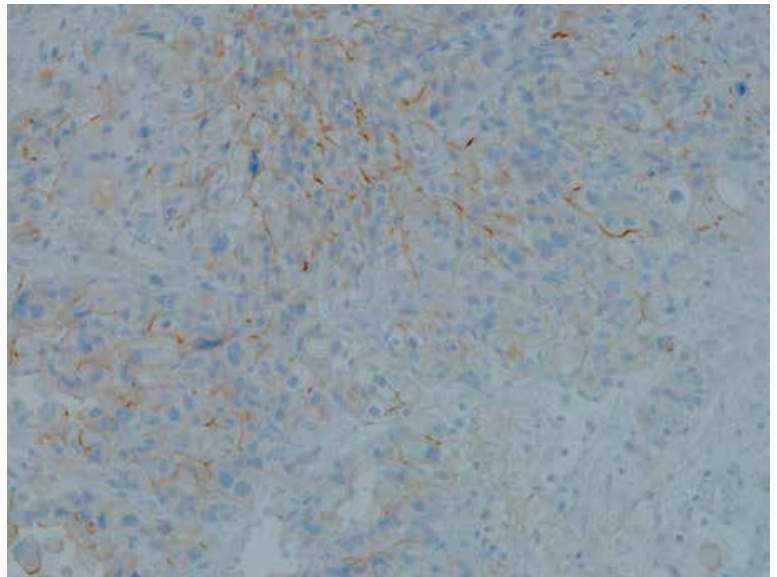

Figure 1. Poorly differentiated rectal adenocarcinoma with EGFR overexpression 

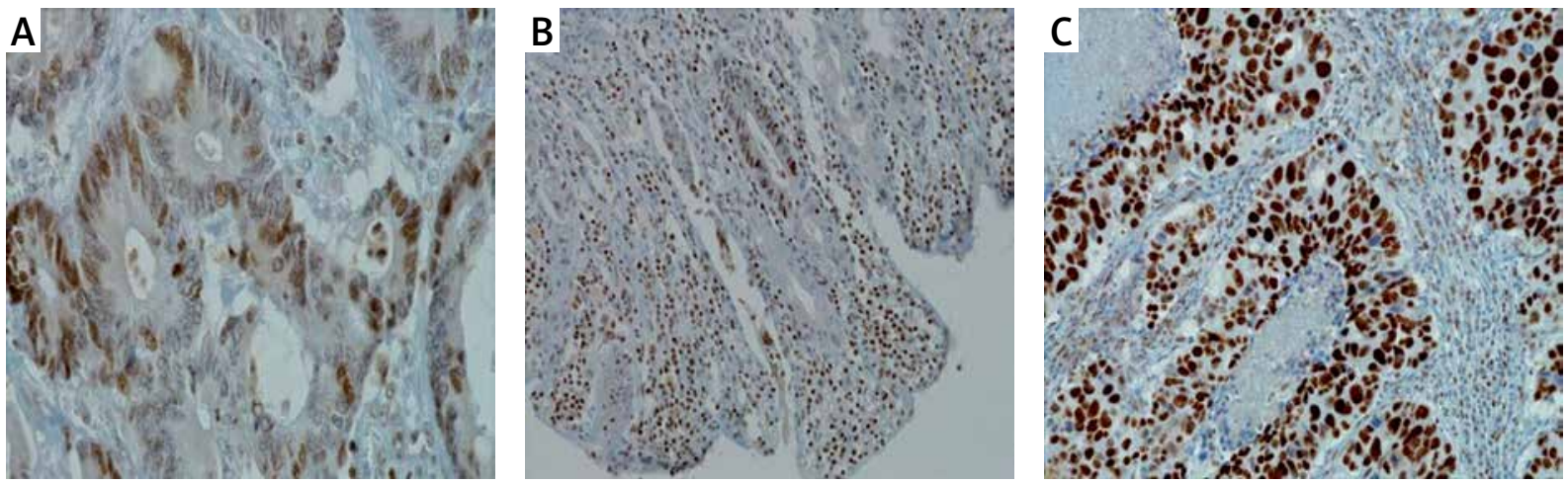

Figure 2. A four-level system describing p21 reactivity: 0 - no reaction, 1 - weak reaction, 2 - moderate reaction and 3 - strong reaction

Systems; Roche Group, Tucson, USA). After fully automated dewaxing and repeated hydration reaction of the samples, the processes of unmasking of the antigen by proteinase $\mathrm{K}\left(37^{\circ} \mathrm{C}, 5 \mathrm{~min}\right)$ were conducted, followed by an incubation period with the primary antibodies (1: 50 dilution, 20 min incubation). The time and the temperature of both the antigen retrieval and the incubation of primary antibodies were strictly in accordance with the manufacturer's recommendations , followed by further routine steps. A universal DAB Ventana detection kit was used. A four-level scale describing the EGFR reactivity was used: 0 - no reaction, 1 - weak reaction, 2 - moderate reaction and 3 -strong reaction (Figure 1).

Classic immunohistochemical tests were applied using antibody (p21WAF1 (clone H252). All performed tests were fully approved with the aim of being applied in vitro. All reactions were carried out using BenchMark XT (Ventana Medical Systems; Roche Group, Tucson), USA). After fully automated dewaxing and reirrigation of samples, the process of antigen unmasking by protease $\mathrm{K}\left(37^{\circ} \mathrm{C}, 5 \mathrm{~min}\right)$ was carried out, followed by incubation with primary antibodies (dilution $1: 50$, incubation time $20 \mathrm{~min}$ ). The temperature of both antigen collection and the incubation of primary antibodies was closely in compliance with the producer's recommendations and then a further standard procedure was followed. A universal DAB detection kit, DAB Ventana ultra-View, was applied. A four-level system describing $p 21$ reactivity was used: 0 - no reaction, 1 weak reaction, 2 moderate reaction and 3 - strong reaction (Figure 2).

All calculations were made using a digital slide analysis using a Hamammatsu NanoZoomer S210 slide scanner (Hamammatsu, Hamamatsu City, Shizuoka Pref. Japan). After the scanning of the entire slide, a digital image analysis was then performed using the Visiopharm membrane application (Visiopharm, Hoersholm, Denmark). The application used allowed us to diversify the intensity of the plasmalemmal reaction and avoid subjectivity.

\section{Statistical analysis}

The collected data were subjected to statistical analysis using the Kaplan-Meier method, log-rank test, Cox proportional hazard model and logistic regression. In the tests, the significance level of 00.5 was adopted for the purposes of statistical inference. In statistical analyses, licensed SAS 9.3 software and Excel were used.

\section{Results}

The follow-up covered a period of 5 years. The endpoints were: OS and RFS understood as local recurrence-free survival and dissemination-free survival. Of the 102 patients studied, 56 survived at the end of the observation period (i.e. 55\%), including one with local recurrence and one with disseminated neoplasm. Forty-six patients did not survive, among whom, 7 had local recurrence and the median survival was 26.6 months, while the median tumor-free survival was 21.6 months.

Survival was analyzed in groups defined according to various criteria.

1. Sex $-66 \%$ of women and $67 \%$ of men survived 3 years. Sex has no significant effect on survival and median survival time (Figure 3).

2. Histopathological diagnosis - the postoperative samples were dominated by adenocarcinoma in 84 patients, i.e. $82.4 \%$, while the rest were mucinous adenocarcinoma. There was no statistically significant difference in the survival of patients with adenocarcinoma and mucinous adenocarcinoma (Figure 4). 3. Histologic tumor grading - the probability of survival decreases with the increase in the degree of histological differentiation. There was no statistically significant difference in the relapse-free survival (without local recurrence and without neoplastic spread) in patients in groups G1, G2 and G3 (Figure 5). 
A

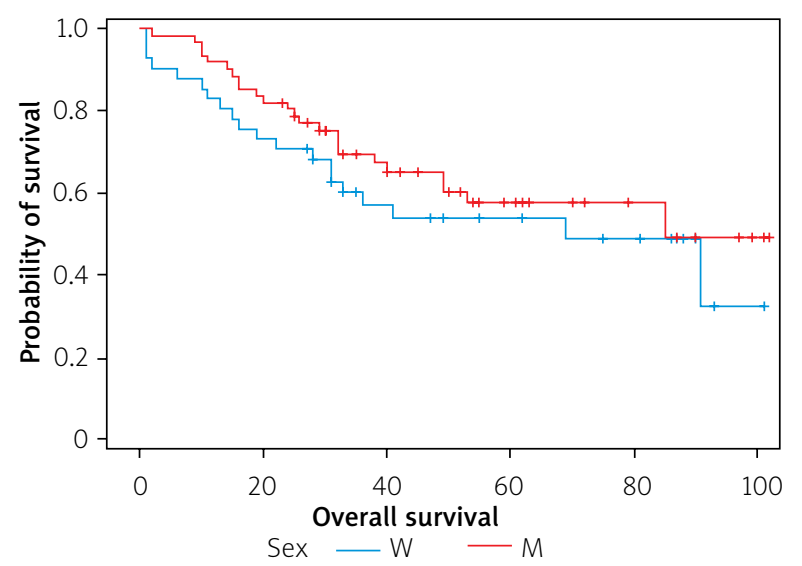

B

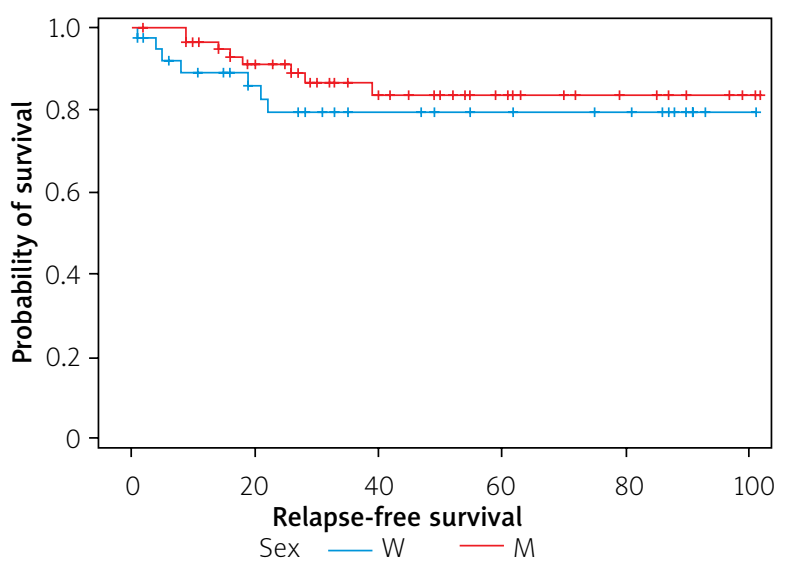

Figure 3. A - Overall survival curves expressed in months in the groups of women $(W)$ and men $(M)$. B - Relapse-free survival curves, expressed in months, in the groups of women (W) and men (M)

A

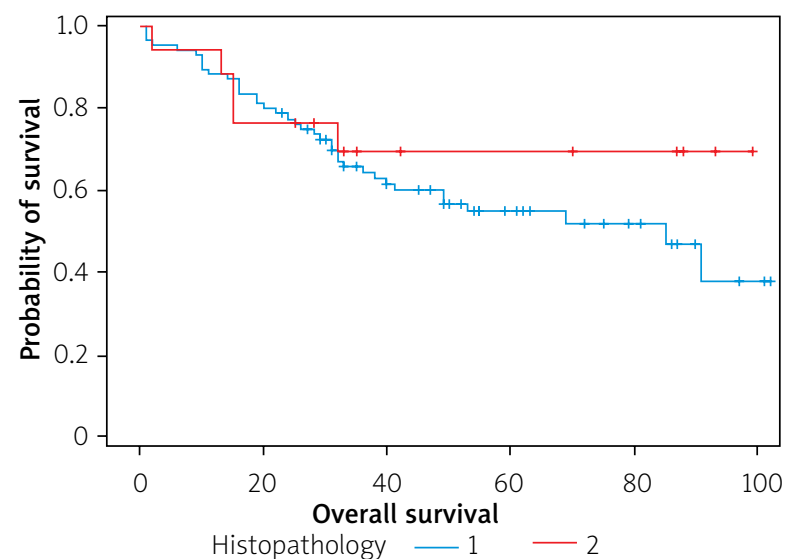

B

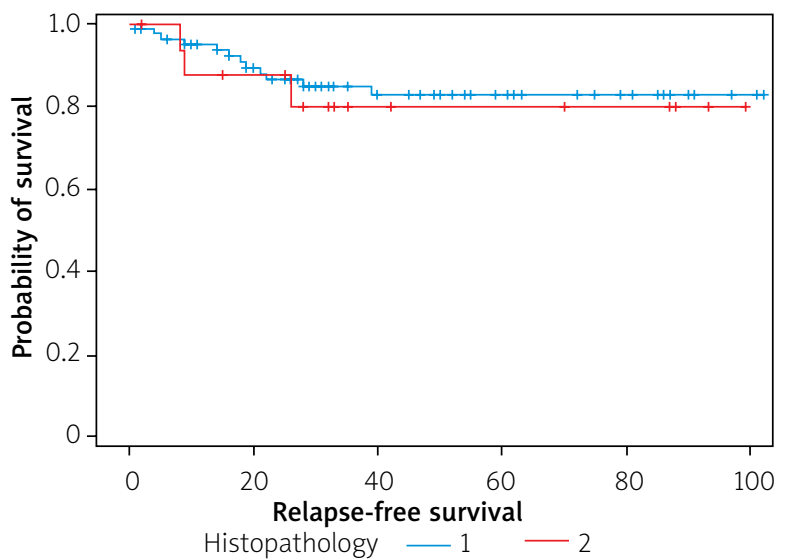

Figure 4. A - The course of the survival curves of patients with histopathological diagnosis: 1 - adenocarcinoma, 2 - mucinous adenocarcinoma. B - Relapse-free survival curves, expressed in months, in the groups of 1 - adenocarcinoma, 2 - mucinous adenocarcinoma

A

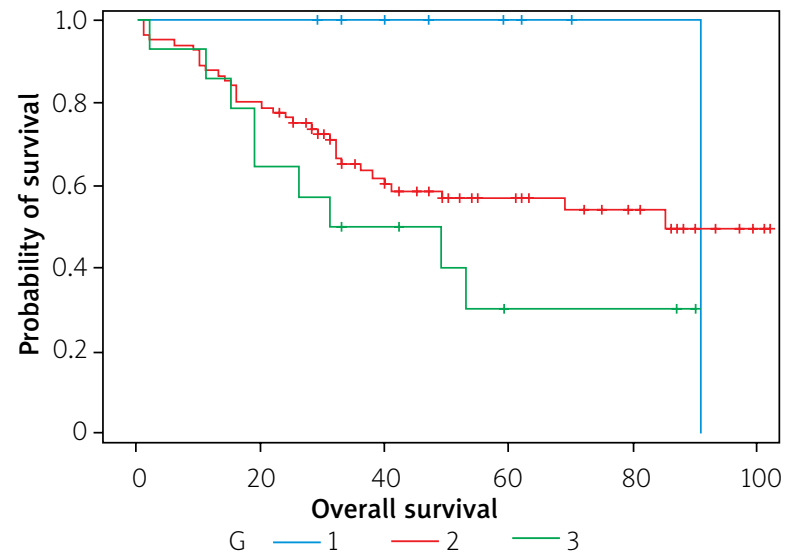

B

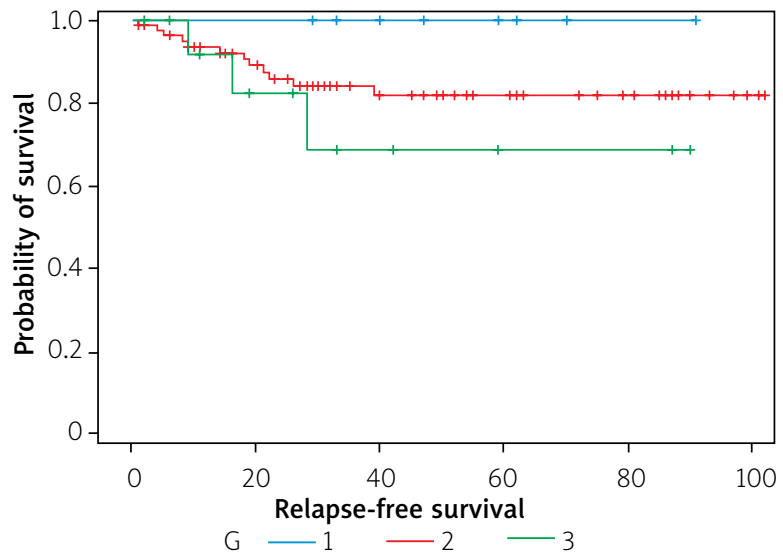

Figure 5. A - Survival curves, B - relapse-free survival curves in months for patients with grading of 1,2 or 3 , respectively 


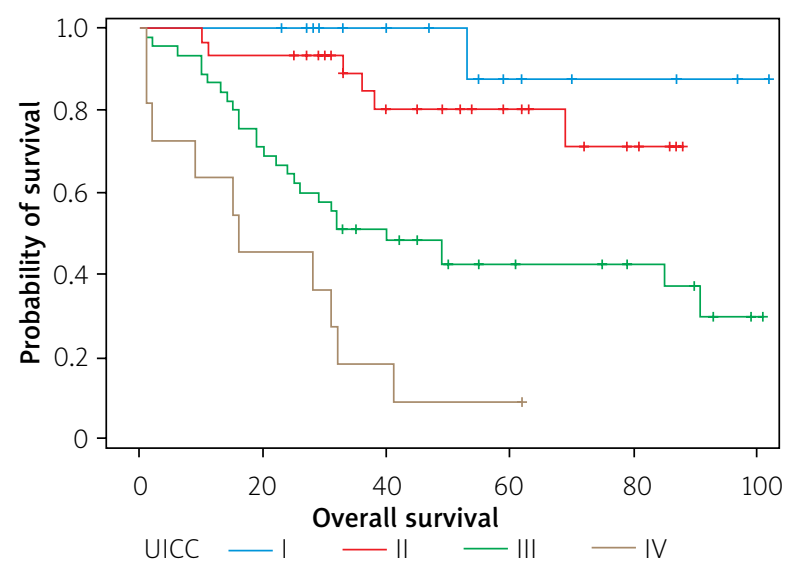

Figure 6. Survival curves, expressed in months, in groups of patients with the UICC stage of the neoplastic disease of I, II, III or IV, respectively

4. UICC stage of cancer disease - the probability of survival decreases with the advancement of cancer disease (Figure 6).

The summary of the multi-stage logistic regression test including BMI, patient age and clinical stage showed that (Table I):

- BMI is not: a significant risk factor for death, does not significantly affect the risk of neoplastic spread and is not a significant risk factor for the recurrence of neoplasm;

- the cancer stage has a statistically significant impact on the risk of death of a patient. With each stage, the risk of death increases by about $10 \%$;

- the age of the patients at the time of surgery indicates a greater risk of death, by about $4 \%$, with each successive year in age.

5. Expression of EGFR - patients with lower EGFR expression live longer than patients with higher EGFR expression (Figure 7).

Higher EGFR intensity increases the risk of death 4 times, the risk of cancer spread 4.6 times, and the risk of cancer recurrence 4.3 times (Table II).

6. Immunoactivity of p21 protein - the probability of survival does not show a statistically significant dependence on the activity of the p21 protein. Relapse-free survival (without local recurrence and cancer spread) of patients who did not reveal p21 immunoactivity $(0 ; 1)$ did not differ significantly from relapse-free survival of patients with p21 hyperimmunoactivity $(2 ; 3)$ (Figure 8 ).

The level of $\mathrm{p} 21$ reactivity is not a significant risk factor for death and is not a significant risk factor for cancer spread.

Table I. Comparison of 2-years RFC with predictive factors (multistep logistic regression test)

\begin{tabular}{lcccc} 
Parameter & Parameter evaluation & OR & $\mathbf{9 5 \% ~ C l}$ & $\boldsymbol{P}$-value \\
\hline BMI & -0.0945 & 0.910 & $0.805-1.028$ & 0.1297 \\
\hline Age & 0.0133 & 1.013 & $0.961-1.069$ & 0.6228 \\
\hline UICC stage & 1.596 & 4.93 & $2.15-11.35$ & 0.0002
\end{tabular}

A

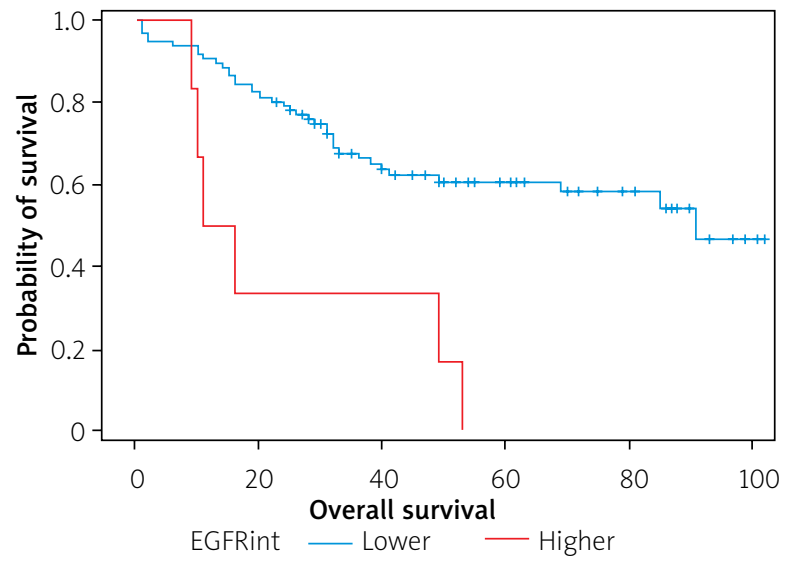

B

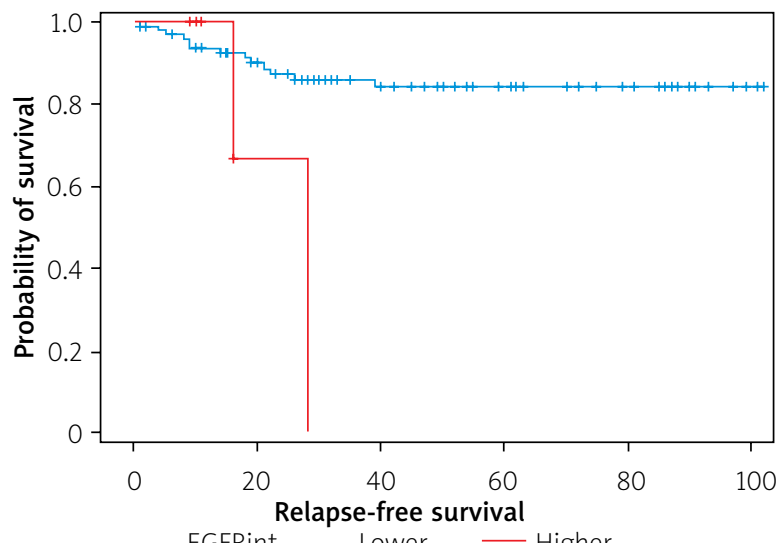

Figure 7. OS curves in log-rank test. A - Low EGFR vs high EGFR expression $p=0.0004$ (low intensity $0-1$, high 2-3), B - relapse-free survival curves in months for patients with lower EGFR intensity (level 0 or 1 ) and higher EGFR intensity (level 2 or 3) $p=0.0408$ 
Table II. Results of using the Cox proportional hazards model with independent variable EGFR expression and death, cancer dissemination as well as cancer recurrence as endpoint events

\begin{tabular}{lcccc} 
Variable & Parameter evaluation & $\chi^{2}$ & Pr. $>\chi^{2}$ & Risk factor \\
\hline EGFR & 1.441 & 10.511 & 0.0012 & 4.228 \\
\hline EGFR & 1.536 & 3.900 & 0.0483 & 4.650 \\
\hline EGFR & 1.467 & 3.508 & 0.0610
\end{tabular}

A

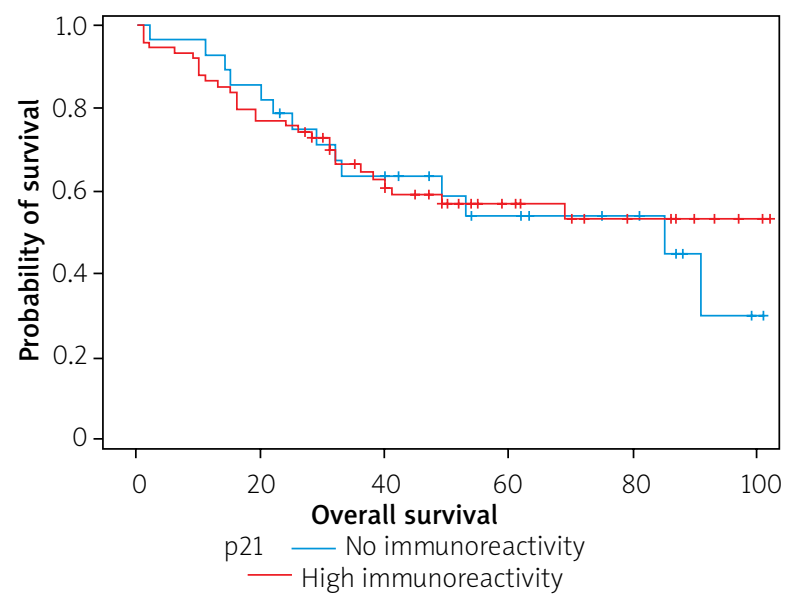

B

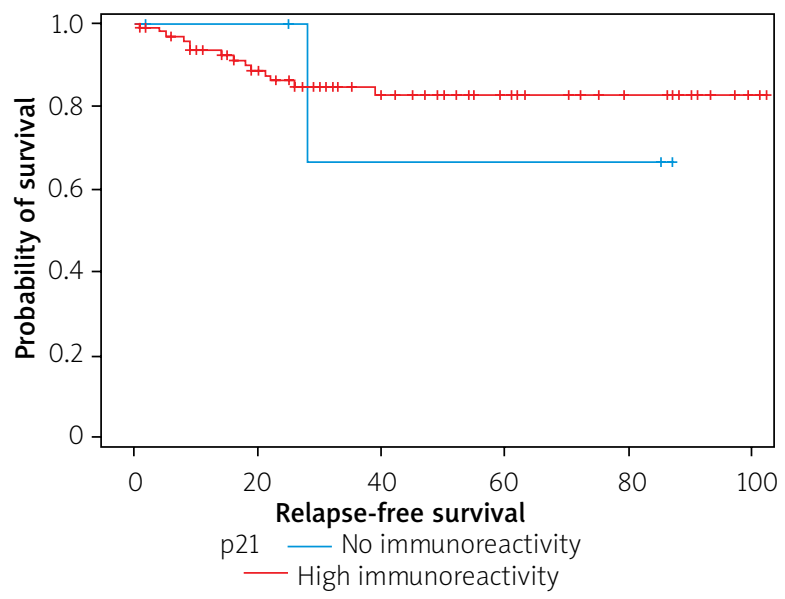

Figure 8. A - Survival curves (expressed in months) for patients who did not show immunoreactivity of p21 (grade 0 and 1 ) and in patients with high immunoreactivity (grade 2 and 3 ). B - Curves of relapse-free survival (expressed in months) for patients with no immunoreactivity of p21 (0.1) and with p21 immunoreactivity (2.3). The relapse-free survival curve (without local recurrence or dissemination) for patients who did not show p21 immunoreactivity does not differ significantly from the survival curve without recurrence for patients with high 21 immunoreactivity ( $p=0.7288$ in the log-rank test)

\section{Discussion}

Although not all publications show that the patient's age is an independent prognostic parameter, it seems reasonable to say that advanced age can be treated as a potential factor that worsens the prognosis, and in older patients poor treatment outcomes could be expected. The median incidence of rectal cancer in women was 65 years of age, and in men it was 66 years of age. The age of the patients at the time of surgery indicates a greater risk of death, by about $4 \%$, with each successive year in age. The causes of the poor prognosis in older people are unclear and difficult to explain. The most probable causes seem to be the state of the immune system and immunity (5) as well as the state of the circulatory system.

There were 41 women and 61 men in the study group of patients. There was no statistically significant difference in survival between women and men in the log-rank test $(p=0.336)$. Similarly, it was found that the probability of relapse-free survival (i.e. without local re- currence and without cancer spread) has no statistically significant dependence on sex $(p=0.8902)$.

The lack of a significant association between the patient's sex and prognosis of the outcome was confirmed by the majority of widely known studies, both older and the latest $[6,7]$. However, some authors found statistically significantly higher rates of overall survival in women $[8,9]$. A possible cause could be the protective effect of pregnancy and childbirth. Perhaps a more important role is played by the structure of the pelvis, as well as adequate radial margins easier to obtain in women and greater oncological radicalism. This is supported by the results of studies by Buhre et al. [10], showing significantly better overall and asymptomatic survival for women, as well as lower rates of locoregional recurrences. This means that the potential reasons for a better prognosis in women can be associated with more effective local control $[5,11]$.

In terms of histopathology, rectal cancer is mainly adenocarcinoma, diagnosed in $98 \%$ of cases. Many 
tumors produce mucin, which is secreted into the intestinal lumen, worsening, according to some research, the prognosis of rectal cancer $[5,11]$. It is estimated that they account for $10-15 \%$ of the total number of colon and rectal cancers. It has also been shown that they are much more common in younger patients, accounting for up to $40 \%$ of cancers before the age of 40 . Okuno et al. [12] and Doglietto et al. [13] confirmed the poor prognosis of mucinogenic carcinomas. Kanemitsu et al. [14] and Nakamura et al. [15] observed significantly poor overall 5-year survival, and Cerottini et al. [16] 10-year survival, noting more frequent recurrences of mucus-secreting tumors and their more extensive spread through continuity. On the other hand, not all publications confirm a significantly poor prognosis for mucinous carcinomas, and Newland et al. [17] recorded even higher survival rates (67\% vs. $59 \%$ ), as did Dent et al. [18].

In the study group, 18 (17.6\%) patients were found to have the tumor producing mucin. There was no statistically significant difference in the survival of patients with a histopathological diagnosis of adenocarcinoma and mucinous adenocarcinoma $(p=0.3635)$. Also, the probability of relapse-free survival (without local recurrence and without neoplastic spread) did not statistically significantly depend on the histopathological diagnosis: adenocarcinoma, mucinous adenocarcinoma ( $p=0.7477$ in the log-rank test). Therefore, it seems justified to say that although mucus secretion does not significantly worsen the treatment results, the histological specificity and correlation with other clinical parameters indicate that these cancers constitute a separate group of neoplasms in terms of developmental biology and spread capacity [5].

It is believed that low-differentiated carcinomas are characterized by more aggressive development and greater growth dynamics. Another hypothesis is related to tumor heterogeneity and assumes the predominance of low-maturity cell clones at the time of intense tumor infiltration and spread. Older studies (Eker [19] and Elliot and Louw [20]) described a significantly higher incidence of low-maturity neoplasms in patients under 30 years of age. Lawday et al. [8] noted a significant predilection of highly differentiated rectal carcinomas to localization in its upper part.

Rectal cancer was found to be $\mathrm{G} 1$ in 8 patients, G2 in 80 patients and $\mathrm{G} 3$ in 14 patients. Statistically significant differences were noted in the survival of patients with $\mathrm{G} 1$, while for $\mathrm{G} 2$ and $\mathrm{G} 3$ grades $p=0.0977$. No statistically significant difference was found in relapse-free survival (without local recurrence and without neoplastic spread) in patients in groups $\mathrm{G} 1, \mathrm{G} 2$ and $\mathrm{G} 3, p=$ 0.3013 .
Most of the patients in the study group, i.e. 74 (72.5\%), were operated on in stage 3 of cancer (tumor-node-metastasis (TNM) 3). Our own research has shown that the probability of survival decreases with the stage of neoplastic disease, according to the UICC classification. The stage has a statistically significant impact on the risk of patient's death $(p<0.0007)$. With the increasing clinical stage of carcinoma, each additional stage increases the risk of death by about $10 \%$ $(\mathrm{HR}=1.100)$.

An increase in EGFR expression was observed in tumors of different locations and was usually associated with a poorer prognosis, increased risk of relapse and a shorter survival rate [21, 22]. However, the reports on the effect of the EGFR hyper-expression on survival in rectal cancer are not conclusive [23]. Part of the results of clinical-pathological studies showed that the EGFR expression is an unfavorable prognostic factor [24, 25]. EGFR expression is observed to varying degrees in solid tumors. According to Herbst et al., EGFR overexpression in rectal cancer occurs in $50-70 \%$ of cancer cases, while according to other researchers, it occurs in about 25$82 \%$. In our study, the EGFR expression at levels 3, 2 and 1 was determined in 33 patients, i.e. $34.68 \%$. Studies to date have not explicitly confirmed the relationship between the EGFR expression and the survival of patients with rectal cancer [26], although the study by Mayer et al. showed that the EGFR expression in more than $50 \%$ of cancer cells is a negative prognostic factor [27]. Moreover, overexpression of EGFR (upregulation) is associated with more aggressive tumor growth, a poorer prognosis and higher resistance to radiation; therefore it can potentially be a useful marker in predicting complete responses [25, 28, 29]. The results obtained in our studies confirmed the above-mentioned reports the probability of survival was higher in patients with a low EGFR intensity, $p=0.0004$. The likelihood of RFS was much higher in patients with a low EGFR expression than in patients with a high EGFR expression ( $p=$ 0.0297). The multicausal analysis showed that the high EGFR intensity increases the risk of death 4-fold $(H R=$ 4.228) and that high EGFR intensity increases the risk of metastases 4.6 times $(H R=4.650)$. The multivariate analysis also showed that high EGFR intensity increases the risk of cancer recurrence 4.3 times $(H R=4.3)$. Interestingly, our study showed that among patients with high EGFR levels, patients with $\mathrm{pT} 4$ predominated $(p=$ $0.0003)$, although some researchers say that the correlation between EGFR overexpression and clinical-pathological parameters is not important [28, 30, 31]. According to them, this may seem only an additional molecular event that worsens the result. In turn, our own research showed that there was no statistically 
significant relationship between EGFR expression and the type of histopathological cancer $(p=0.1965)$. Based on the results of clinical observations, EGFR expression was found to be an adverse prognostic factor. The EGFR blocked by the monoclonal antibody entails the inhibition of many biological signaling pathways, often worsening the result $[28,31]$.

p21 participates in the regulation of numerous normal cellular functions, including proliferation, differentiation and apoptosis. A very close connection of p21 with TP53 suppressor gene mutation led to the conclusion about its clinical usefulness in outcome prognosis. In assessing the clinical value of p21, the nuclear accumulation of this protein was usually determined qualitatively, with its presence in tumor cells between $36 \%$ and $71 \%$, and even up to $80 \%$ of cases $[32,33$.

In our study, we used a repeatable 4 tiered scale supported by digital analysis to separate a weak and evident reaction. Moreover, the applied solution allowed us to avoid natural subjectivism. The highest activity of p21was detected in 74 patients, i.e. $72.5 \%$. In 5 patients, i.e. almost 5\%, p21 activity was not detected.

Survival curves in four groups of patients, based on the p21 expression level, show no statistically significant differences ( $p=0.6453$ in the log-rank test). The survival curve for patients who did not express p21 (0) did not significantly differ from the survival curve for patients with p21 overexpression $(1 ; 2 ; 3)(p=0.2205)$. Observation of the course of treatment for relapse covered the period up to 102 months after surgery. In the literature, there was no clear relationship between the development of tumor recurrence and the appearance of p21ras protein expression [33, 34]. In our study, it was also found that the level of p21 did not affect cancer recurrence. Some researchers have detected such a relationship, although to a limited extent, for example, concerning rectal cancers or a group of patients in stage $A$ and B, according to Dukes [35]. There was also no clear correlation between frequency and RFS and the presence of p21 expression. This conclusion was confirmed by our study, as there were no statistically significant differences in RFS between groups of patients in expression groups 0, 1, 2, 3, $p$-value $=0.9650$ in the log-rank test. Based on the data presented, we suggest a lack of prognostic significance of p21 in patients with sporadic rectal adenocarcinoma; however, the immunohistochemical assessment of the presence of p21ras may be useful in assessing tumor biology, especially in combination with other molecular preparations [33, 36, 37].

In our research among patients with p21 activity level 0,1 or 2, the proportions of patients for whom the time from surgery to death or the end of observation was below 2 years, between 2 and 5 years, or over
5 years, were at a similar level. Cox proportional hazard model application with binary independent variable assuming a value of 0 when p21 level is 0 or 1 , and a value of 1 when p21 level is 2 or 3 , leads to the conclusion that p21 expression level is not a significant risk factor for death $(p=0.7842)$, and is also not a significant risk factor for tumor dissemination ( $p=0.9426)$. Our data presented here do not allow us to assign a greater significance to p21 expression in detecting and monitoring the course of rectum cancer treatment.

\section{Conclusions}

Overall survival and relapse-free-survival do not depend on sex, histopathological diagnosis, histologic tumor grading, or BMI. Patients with lower EGFR expression live longer than patients with higher EGFR expression. The probability of relapse-free survival was significantly higher in patients with lower EGFR expression than in patients with higher EGFR expression. Higher EGFR intensity increases the risk of death 4 times, the risk of cancer spread 4.6 times, and the risk of cancer recurrence 4.3 times. The level of p21 reactivity is not a significant risk factor for death and is not a significant risk factor for cancer spread.

\section{Acknowledgments}

Moreover, the authors are very grateful to Antoinette Urbaniak MD, PhD, Head of Private Pathology Department, for her cooperation.

Project financed under the research work no. SMGR. RN.20.177.

\section{Conflict of interest}

The authors declare no conflict of interest.

\section{References}

1. Kucejko RJ, Holleran TJ, Stein DE, Poggio JL. How soon should patients with colon cancer undergo definitive resection? Dis Colon Rectum 2020; 63: 172-82.

2. Grass F, Behm KT, Duchalais E, et al. Impact of delay to surgery on survival I stage I-III colon cancer. Eur J Surg Oncol 2020: 46: 455-61.

3. Dharwadkar P, Greenan G, Singal AG, Murphy CC. Is colorectal cancer in patients younger than 50 years of age the same disease as in older patients? Clin Gastroenterol Hepatol 2021; 19: 192-4.e3.

4. Platell C, Barwood N, Dorfmann G, Makin G. The incidence of anastomotic leaks in patients undergoing colorectal surgery. Colorect Dis 2007; 9: 71-9.

5. Szpon $Ł$, Stal A, Zawadzki M, et al. K-ras gene mutation as an early prognostic marker of colon cancer. Pol Przegl Chir 2016; 88: 15-9.

6. Lydrup ML, Hogkind P. Gender aspects of survival after surgical treatment for rectal cancer. Colorectal Dis 2015; 17: 390-6. 
7. Nara A, Udagawa M, Onishi I, et al. Long-term survival of a patient with advanced recurrent rectal cancer treated with a multidisciplinary therapy including five operations - a case report. Gan To Kagaku Ryoho 2020; 47: 114-6.

8. Lawday S, Ricciardi E, Bethune R, McDermott F. Response to Preoperative geriatric assessment and tailored interventions in frail older patients with colorectal cancer: a randomized controlled trial. Colorectal Dis 2018; 20: 350.

9. Godwin J, Brown C. Some prognostic factors in survival of patients with cancer of the colon and rectum. J Chronic Dis 1975 28: 441-54.

10. Buhre L, Mulder N, de Ruiter AJ, et al. Effect of extent of anterior resection and sex on disease-free survival and local recurrence in patients with rectal cancer. Br J Surg 1994; 81: 1227-9.

11. Zaręba KP, Zińczuk J, Dawidziuk T, et al. Can factors that influence nodal dissemination in patients with colorectal cancer be identified? Own experience. Gastroenterology Rev 2020 15: 247-52.

12. Okuno M, Ikehara T, Nagayama $M$, et al. Mucinous colorectal carcinoma: clinic pathology and prognosis. Am Surg 1988; 54 681-5.

13. Doglietto G, Bellantone R, Sofo L. Mucinous carcinoma of the colorectum: clinical features, pathology and prognosis. Coloproctology 1991; 1: 51.

14. Kanemitsu Y, Kato T, Hirai T, et al. Survival after curative resection for mucinous adenocarcinoma of the colorectum. Dis Colon Rectum 2003; 46: 160-7.

15. Nakamura Y, Shida D, Tanabe T, et al. Prognostic impact of preoperatively elevated and postoperatively normalized carcinoembryonic antigen levels following curative resection of stf f cxaa age I-III rectal cancer. Cancer Med 2020; 9: 653-62.

16. Cerottini J, Caplin S, Pampallona S, Givel JC. Prognostic factors in colorectal cancer. Oncol Rep 1999; 6: 409-14.

17. Newland R, Dent O, Lyttle MN, et al. Pathologic determinants of survival in colorectal cancer with lymphnode metastases: multivariate analysis of 579 patients. Cancer 1994; 73: 2076-82.

18. Dent OF, Bokey L, Chapnis PH, et al. Trends in short-term outcomes after resection of colorectal cancer: 1971-2013. ANZ J Surg 2017; 87: 39-43.

19. Eker R. Some prognostic factors for carcinoma of the colon and rectum. Acta Chir Scand 1963; 126: 636-56.

20. Elliot M, Louw T. A 10 year study at Grotte Schuur Hospital with particular reference to patients under 30 years of age. Br J Surg 1979; 66: 621-4.

21. Feigelson HS, Zeng C, Pawloski PA, et al.; CERGEN Study Team. Does KRAS testing in metastatic colorectal cancer impact overall survival? A comparative effectiveness study in a population-based sample. PLoS One 2014; 9: e94977.

22. Formica V, Roselli M. Targeted therapy in first line treatment of RAS wild type colorectal cancer. World J Gastroenterol 2015; 21: 2871-4.

23. Skonieczna K, Jawień A, Marszałek A, Grzybowski T. Mitogenome germline mutations and colorectal cancer risk in Polish population. Arch Med Sci 2020; 16: 366-73

24. Gupta S, Sun H, Yi S, et al. Molecular markers of carcinogenesis for risk stratification of individuals with colorectal polyps: a case-control study. Cancer Prev Res (Phila) 2014; 7 : 1023-34.
25. Herbst RS, Maddox AM, Rothenberg ML, et al. Selective oral epidermal growth factor receptor tyrosine kinase inhibitor ZD1839 is generally well-tolerated and has activity in non-small cel lung cancer and other solid tumors: results of a phase I trial. J Clin Oncol 2002; 20: 3815-25.

26. Hohla F, Winder T, Greil R, et al. Targeted therapy in advanced metastatic colorectal cancer: current concepts and perspectives. World J Gastroenterol 2014; 20: 6102-12.

27. Mayer A, Takimoto M, Fritz E, et al. The prognostic significance of proliferating cell nuclear antygen, epidermal growth factor receptor, and mdr gene expression in colorectal cancer. Cancer 1993; 71: 2454-60.

28. Kozłowska-Geller MA, Lewitowicz P, Głuszek S. How does overexpression affect the development and treatment of rectal cancer? Stud Med 2018; 34: 337-41.

29. Jeong WJ, Cha PH, Choi KY. Strategies to overcome resistance to epidermal growth factor receptor monoclonal antibody therapy in metastatic colorectal cancer. World J Gastroenterol 2014; 20: 9862-71.

30. Karantanos T, Theodoropoulos G, Pektasides D, Gazouli M. Clock genes: their role in colorectal cancer. World J Gastroenterol 2014; 20: 1986-92.

31. Kishiki T, Ohnishi H, Masaki T, et al. Impact of genetic profiles on the efficacy of anti-EGFR antibodies in metastatic colorectal cancer with KRAS mutation. Oncol Rep 2014; 32: 57-64.

32. Karantanos T, Theodoropoulos G, Pektasides D, Gazouli M. Clock genes: their role in colorectal cancer. World Gastroenterol 2014; 20: 198692

33. Kozłowska-Geller MA, Głuszek SZ, Lewitowicz P. P21 is not a prognostic marker for rectal cancer - five-year follow up study of rectal cancer in stages I-IV. Contemp Oncol 2020; 24: 247-51.

34. Zhang S, Sui L, Zhuang J, et al. ARHGAP 24 regulates cel ability and apoptosis of colorectal cancer cells via the regulation of p53. Oncol Lett 2018; 16: 3517-24.

35. Neumann JH, Jung A, Kirchner Y. Molecular pathology of colorectal cancer. Pathologe 2015; 36: 137-44.

36. Vaish V, Rana C, Piplani H, et al. Sulindac and Celecoxib regulate cel cycle progression by $\mathrm{p} 53 / \mathrm{p} 21$ up regulation to induce apoptosis during initial stages of experimental colorectal cancer. Cell Biochem Biophys 2014; 68: 301-19.

37. Zhang Y, Geng L, Talmon G, Wang J. MicroRNA-520 g confers drug resistance by regulating p21 expression in colorectal cancer. J Biol Chem 2015; 290: 6215-25.

Received: 28.01.2021

Accepted: 13.02.2021 